11,05

\title{
Квазиодномерные модели Изинга с дефектами типа „случайное локальное поле“: фаза Имри-Ма в пространствах размерности, большей нижней критической
}

\author{
(C) А.А. Берзин ${ }^{1}$, А.И. Морозов ${ }^{2}$, А.С. Сигов ${ }^{1}$ \\ ${ }^{1}$ МИРЭА - Российский технологический университет, \\ Москва, Россия \\ ${ }^{2}$ Московский физико-технический институт (национальный исследовательский университет), \\ Долгопрудный, Московская обл., Россия \\ E-mail: assigov@yandex.ru
}

Поступила в Редакцию 11 сентября 2020 г.

В окончательной редакции 11 сентября 2020 г.

Принята к публикации 13 сентября 2020 г.

\begin{abstract}
Исследована фазовая диаграмма „температура-концентрация дефектов“ квазиодномерных моделей Изинга с дефектами типа „случайное локальное поле“. Изучено противоборство тенденции к возникновению дальнего порядка вследствие слабого взаимодействия между одномерными спиновыми цепочками и тенденции к образованию фазы Имри-Ма, в которой параметр порядка следует за флуктуациями случайного поля, созданного дефектами. Показана возможность возникновения фазы Имри-Ма в ситуации, когда размерность пространства превосходит нижнюю критическую размерность. Рассмотрен вопрос о наличии дальнего порядка в модели Изинга со случайными полями в пространстве с критической размерностью $d_{l}=2$.
\end{abstract}

Ключевые слова: дефекты типа „случайное локальное поле“, квазиодномерная модель Изинга, фазовая диаграмма, фаза Имри-Ма.

DOI: 10.21883/FTT.2021.01.50411.195

\section{1. Введение}

При температуре, отличной от абсолютного нуля, дальний порядок в цепочке изинговских спинов отсутствует. С понижением температуры в бездефектной системе одномерный радиус корреляции спинов экспоненциально растет. При достижении им критического размера в квазиодномерной системе становится существенным слабое обменное взаимодействие спинов, принадлежащих соседним спиновым цепочкам. Происходит кроссовер от одномерного поведения к $d$-мерному $(d \geq 2)$, и в системе возникает дальний порядок.

В квазиодномерной системе изинговских спинов с дефектами типа „случайное локальное поле“ может возникнуть другой сценарий поведения системы. При понижении температуры $T$ ниже некоторого значения $T^{*}$ происходит переход от динамических флуктуаций параметра порядка к его статическим флуктуациям, которые следуют за флуктуациями случайного поля дефектов. В результате возникает неупорядоченное состояние Имри-Ма, которое сохраняется вплоть до абсолютного нуля температуры.

Реализуется первый или второй сценарий, зависит от соотношения между характерным масштабом статических флуктуаций, зависящим от концентрации дефектов, и критическим радиусом корреляции, который определяется межцепочечным взаимодействием. Настоящая работа посвящена нахождению областей физических параметров, отвечающих указанным сценариям и построению фазовой диаграммы „температура-концентрация дефектов“" рассматриваемой системы.

\section{2. Энергия системы классических изинговских спинов}

Энергия обменного взаимодействия классических изинговских спинов, образующих $d$-мерную квадратную решетку, в приближении взаимодействия ближайших соседей равна

$$
w_{e x}=-\frac{1}{2} J \sum_{i, \delta} \sigma_{i} \sigma_{i+\delta},
$$

где $J>0$ - обменный интеграл, проекция спина на легкую ось $\sigma_{i}$ принимает значение \pm 1 , суммирование по $i$ ведется по всей решетке спинов, а по $\delta$ - по ближайшим к данному спину соседям.

Энергия взаимодействия спинов со случайными локальными полями дефектов равна

$$
w_{\text {def }}=-\sum_{l} \sigma_{l} h_{l},
$$

суммирование ведется по случайно расположенным в узлах решетки дефектам, $h_{l}$ - локальное поле $l$-того 
дефекта, которое случайным образом принимает значение $\pm h_{0}$.

\section{3. Наличие дальнего порядка в двумерной модели Изинга с дефектами типа „случайное локальное поле“}

Хорошо известно, что нижняя критическая размерность модели Изинга с короткодействующим обменным взаимодействием и случайными полями (random-field Ising model) равна двум [1-3]. Вместе с тем в вопросе о существовании дальнего порядка при размерности пространства $d_{l}=2$ существуют противоположные точки зрения. В работах $[4,5]$ на этот вопрос дан отрицательный ответ, в то время как в работах [6,7] было показано, что в области слабых случайных полей при нулевой температуре дальний порядок имеет место. Следует отметить, что авторы, как правило, рассматривают квадратную решетку изинговских спинов, в которой случайное поле существует на каждом узле решетки, а его величина описывается гауссовским распределением.

Рассмотрим более реалистическую модель, в которой случайные поля создаются дефектами. Приведем для этого случая аргументы Имри и Ма [1]. Среднее (в расчете на одну ячейку) поле дефектов в области с линейным размером $L$ (в единицах постоянной решетки), обусловленное статистическими флуктуациями числа дефектов с противоположным направлением случайного поля, равно $h_{0} c^{1 / 2} / L^{d / 2}$, где $c \ll 1$ безразмерная концентрация дефектов (их число в расчете на элементарную ячейку) [8]. Выигрыш в энергии за счет возникновения фазы Имри-Ма, в которой параметр порядка в каждой области размером $L$ направлен по среднему полю, составляет в расчете на одну ячейку величину

$$
w_{\mathrm{I}-\mathrm{M}} \approx-\frac{h_{0} c^{1 / 2}}{L^{d / 2}}
$$

Проигрыш в энергии за счет возникновения резких доменных стенок на границах областей составляет в расчете на одну ячейку величину

$$
w_{e x} \approx \frac{2 d J}{L} .
$$

Таким образом, при $d<2$ фаза Имри-Ма становится энергетически более выгодной при достаточно большом значении $L$. Оптимальное значение $L^{*}$, отвечающее минимуму суммарной энергии $w_{\mathrm{I}-\mathrm{M}}+w_{\text {ex }}$, и само минимальное значение энергии $w^{*}$ равны для $d=1$ соответственно

$$
\begin{gathered}
L^{*} \approx \frac{16 J^{2}}{c h_{0}^{2}}, \\
w^{*} \approx-\frac{c h_{0}^{2}}{8 J} .
\end{gathered}
$$

При $d=2$ зависимость $w_{\mathrm{I}-\mathrm{M}}$ и $w_{e x}$ от $L$ одинакова. Но при $J^{2} \gg c h_{0}^{2}$ состояние с дальним порядком оказывается основным. Это простое энергетическое рассмотрение находится в согласии с выводами работ $[6,7]$. Следовательно, фаза Имри-Ма отсутствует в двумерной системе, и следовательно, во всех квазидвумерных системах в пространствах с $d>2$.

Перейдем к рассмотрению возможности существования фазы Имри-Ма в квазиодномерной системе со слабым взаимодействием между спиновыми цепочками.

\section{4. Квазиодномерная модель Изинга с дефектами типа „случайное локальное поле“}

Пусть индекс $m$ нумерует параллельные спиновые цепочки, образующие в перпендикулярном срезе $d$-1-мерную квадратную решетку $(d \geq 2)$, а индекс $i-$ спины вдоль данной цепочки. Тогда энергия взаимодействия спинов имеет вид

$$
w_{e x}=-J_{\|} \sum_{i, m} \sigma_{i, m} \sigma_{i+1, m}-\frac{1}{2} J_{\perp} \sum_{i, m, \delta} \sigma_{i, m} \sigma_{i, m+\delta},
$$

суммирование по $i$ и $m$ ведется по всей решетке спинов, а по $\delta$ - по ближайшим к данной соседним цепочкам. Обменное взаимодействие соседних спинов, принадлежащих одной цепочке, $J_{\|}>0$ намного превосходит таковое между соседними спинами, принадлежащими разным цепочкам $J_{\perp}>0$. Энергия взаимодействия спинов со случайными локальными полями дефектов задается формулой (2), если считать, что индекс $l$ задает пару индексов $i_{l}, m_{l}$.

Температура кроссовера от одномерного к $d$-мерному поведению и возникновения дальнего порядка в приближении среднего поля находится из условия обращения восприимчивости системы спинов в бесконечность [9] или из условия равенства температуры и энергии взаимодействия коррелированного участка спинов, задаваемого одномерным радиусом корреляции $r_{\|}$, с молекулярным полем

$$
T \approx{ }_{z} J_{\perp} r_{\|},
$$

где $z$ - число спиновых цепочек, ближайших к данной. В нашей модели $z=2(d-1)$. Согласно [9]

$$
r_{\|}=\frac{1}{2} \exp \left(\frac{2 J_{\|}}{T}\right)
$$

Подставляя выражение для $r_{\|}$в формулу (8) и решая получившееся уравнение самосогласования для $T$ методом итераций, получаем для температуры ферромагнитного перехода в бездефектной системе [9]

$$
T_{c} \approx \frac{2 J_{\|}}{\ln \left(\frac{4 J_{\|}}{z J_{\perp}}\right)}
$$




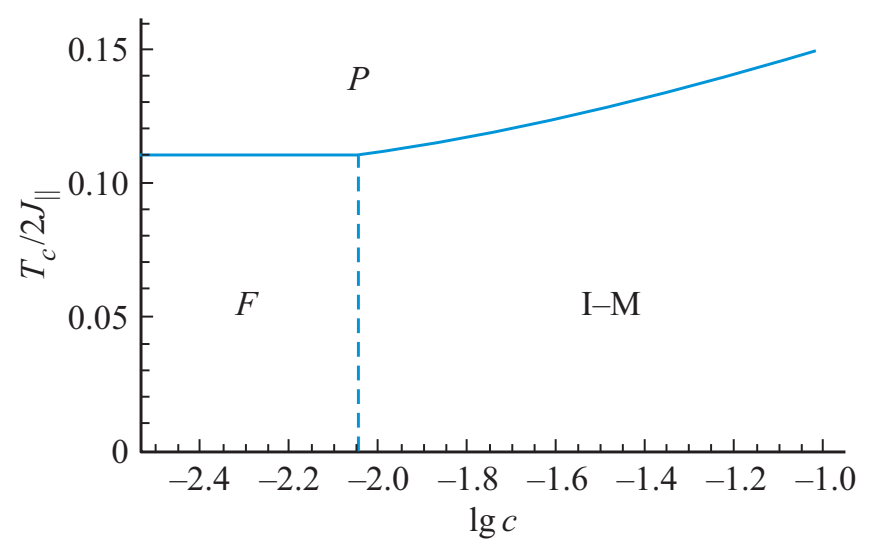

Фазовая диаграмма квазиодномерной модели Изинга с дефектами типа „случайное локальное поле“ при $z=4$, $h_{0}^{2} / J_{\|}^{2}=10^{-1}$ и $J_{\perp} / J_{\|}=10^{-4}: P-$ парамагнитная фаза, $F-$ ферромагнитная фаза, I-M - фаза Имри-Ма.

Поправка к энергии основного состояния невзаимодействующих бездефектных спиновых цепочек за счет взаимодействия между цепочками и возникновения $d$-мерного дальнего порядка в расчете на одну ячейку составляет величину

$$
w_{d}=-\frac{z J_{\perp}}{2} .
$$

Дефекты типа „случайное локальное поле“ при $2 J_{\|}>h_{0}$ не изменяют энергии упорядоченного состояния в силу случайного знака поля дефекта.

Если же в системе реализуется фаза Имри-Ма, то корреляция между цепочками оказывается нарушенной, и поправка к энергии основного состояния невзаимодействующих бездефектных спиновых цепочек за счет взаимодействия со случайными полями описывается формулой (6) с $J \equiv J_{\|}$.

Таким образом, для возникновения фазы Имри-Ма необходимо, чтобы

$$
\left|w^{*}\right|>\left|w_{d}\right|
$$

откуда получаем условие для концентрации дефектов

$$
c>\frac{4 z J_{\perp} J_{\|}}{h_{0}^{2}} .
$$

Для $c \sim 10^{-2}$ и $h_{0}^{2} / J_{\|}^{2} \sim 10^{-1}$ это дает условие $J_{\perp} / J_{\|} \lesssim 10^{-4}$. В случае столь слабого обменного взаимодействия существенным может стать дипольдипольное взаимодействие между спинами. Для возникновения фазы Имри-Ма его величина также должна не превосходить $10^{-4} J_{\|}$.

Следовательно, фаза Имри-Ма может наблюдаться только в случае очень слабого взаимодействия между спиновыми цепочками.

Температура ее возникновения, то есть температура перехода от динамических флуктуаций параметра порядка к его статическим флуктуациям, индуцированным крупномасштабными флуктуациями случайного поля дефектов, происходит при температуре $T^{*}$, которая находится из условия $L^{*}=r_{\|}[10]$ :

$$
T^{*} \approx \frac{2 J_{\|}}{\ln \left(\frac{32 J_{\|}^{2}}{c h_{0}^{2}}\right)} \text {. }
$$

Легко видеть, что условие (12) эквивалентно условию $T^{*}>T_{c}$.

Фазовая диаграмма квазиодномерной модели Изинга с дефектами типа „случайное локальное поле“ в переменных „температура-концентрация дефектов“ изображена на рисунке.

Хорошо известно [3], что немагнитная примесь замещения или вакансия в двухподрешеточном коллинеарном антиферромагнетике, находящемся во внешнем магнитном поле, представляет собой дефект типа „случайное локальное поле“. Величина локального поля прямо пропорциональна индукции приложенного коллинеарно намагниченностям подрешеток магнитного поля. Состояние Имри-Ма может возникнуть в коллинеарной фазе квазиодномерного антиферромагнетика в случае выполнения условия (13).

\section{5. Выводы}

Сформулируем основные выводы работы.

1. В случае малой концентрации дефектов типа „случайное локальное поле“ для нижней критической размерности $d_{l}=2$ в модели с одинаковым по величине обменным взаимодействием со всеми ближайшими соседями основным является состояние с дальним порядком.

2. В случае квазиодномерной модели Изинга с дефектами типа „случайное локальное поле“ - возможно возникновение неупорядоченной фазы Имри-Ма несмотря на то, что низкотемпературное поведение системы является эффективно $d$-мерным с $d \geq 2$, то есть размерность пространства может превосходить нижнюю критическую размерность, полученную для случая, когда величина обменного взаимодействия со всеми ближайшими соседями одинакова.

3. Для возникновения фазы Имри-Ма необходимо, чтобы обмен между спинами соседних спиновых цепочек был на четыре (и более) порядков слабее обмена спинов в цепочке.

\section{Финансирование работы}

Работа поддержана Российским научным фондом (соглашение № 17-12-01435-П).

\section{Конфликт интересов}

Авторы заявляют, что у них нет конфликта интересов. 


\section{Список литературы}

[1] Y. Imry, S.-k. Ma. Phys. Rev. Lett. 35, 1399 (1975).

[2] J. Imbrie. Phys. Rev. Lett. 53, 1747 (1984).

[3] Вик.С. Доценко. УФН 165, 481 (1995)

[4] M. Aizerman, J. Wehr. Phys. Rev. Lett. 62, 2503 (1989).

[5] L. Leuzzi, G. Parizi. Phys. Rev. B 88, 224204 (2013).

[6] C. Frontera, E. Vives. Phys. Rev. E 59, R1295 (1999).

[7] S. Sinha. Phys. Rev. E 87, 022121 (2013).

[8] А.И. Морозов, А.С. Сигов. Письма в ЖЭТФ 90, 818 (2009).

[9] D.J. Scalapino, Y. Ymry, P. Pincus. Phys. Rev. B 11, 2042 (1978).

[10] А.А. Берзин, А.И. Морозов, А.С. Сигов. ФТТ 62, 281 (2020).

Редактор Т.Н. Василевская 